\title{
ESTADO ACTUAL DE LA EXPLOTACION DE LOS PRINCIPALES PECES ORNAMENTALES DE LA AMAZONIA PERUANA
}

Tello M artín Salvador ( $\left.{ }^{*}\right)$

Cánepa la Serna J.R $\left(^{* *}\right)$

\section{INTRODUCCION}

El ecosistema amazónico está conformado por innumerables ríos, quebradas y lagunas, cuyas aguas, por sus condiciones físicas, químicas y biológicas, son lugares adecuados para la vida y desarrollo de las poblaciones ícticas. Se calcula la existencia de aproximadamente 2,000 especies (Sioli, 1975), citado por Bonetto, 1981, de las cuales se han identificado hasta el momento 625 (Ortega y $V$ an, 1986), incluídos los peces de consumo humano y ornamental.

Los peces amazónicos peruanos, por su variedad, abundancia, belleza y alto valor en el mercado internacional, han dado origen a un negocio sumamente lucrativo que, en la actualidad, representa movimientos económicos del orden de millares de dólares (USA), generando significativos ingresos al fisco por concepto de divisas. Es por este motivo que la exportación de peces ornamentales está considerada como una actividad socio-económica muy importante en la Región de Loreto, de la cual dependen numerosas familias (5,000 personas aproximadamente); sin embargo, poco 0 nada se ha hecho para conocer su real estado de explotación.

EI presente trabajo tiene como objetivo analizar esta problemática, como una contribución al conocimiento de la bioecología y del potencial del recurso pesquero ornamental para lograr una administración adecuada y paralelamente manejar y explotar en forma sostenida dicho recurso.

\section{ANTECEDENTES}

Hasta el momento se han efectuado dos estudios importantes sobre esta actividad económica. En 1969 se nombró una comisión técnica para llevar a cabo un análisis de la comercialización, exportación y gravámenes de peces, animales vivos y plantas acuáticas. En este informe se hace una descripción completa de todas las etapas involucradas en la explotación de peces ornamentales, desde la captura hasta el

\footnotetext{
* Director Ejecutivo de Biología (IIAP)

** Ex-Investigador IIAP
} 
embarque final de los peces. Del mismo modo se presenta un análisis detallado de la problemática pesquera ornamental.

En diciembre de 1984, el Instituto de Investigación de la A mazonía Peruana (IIAP), con la participación de especialistas de otros sectores, preparó y presentó un diagnóstico donde se incluyó un análisis situacional de las pesquerías de consumo humano y ornamental de la región amazónica (L oreto - U cayali).

\section{MATERIAL Y METODO}

Este estudio se ha desarrollado en la dudad de Iquitos, Región del A mazonas, debido a que es la principal abastecedora de peces ornamentales del mercado nacional e internacional.

La metodología empleada se diseñó tomando como base el Diagnóstico Pesquero (1984) y consistió básicamente en lo siguiente:

- Elaboración de un esquema de trabajo tentativo.

- Diseño de un formato para la recopilación de datos.

- Visitas y encuestas a los acuarios, pescadores, exportadores, empresas y organismos públicos relacionados con este sector.

- Tabulación, procesamiento y análisis de la información.

En cuanto a la lista preliminar de especies por familias genéricas, se ha tomado como referencia los trabajos de Fowler (1945 -1954), A xelrod (1980-1985), Ortega y Vari (1986) e información del Sr. Porflrio Dávila, técnico de la Dirección Regional de Pesquería.

\section{RESULTADOS}

Como cuestión previa al desarrollo del documento, es conveniente mencionar que los resultados indicados hacen referencia en forma general a la parte descriptiva de esta pesquería: sin embargo, se analiza con mayor amplitud lo correspondiente al recurso íctico ornamental. A simismo, al desarrollar el Capítulo "Volumen de Extracción", se hace referencia específicamente a los volúmenes exportados 0 comercializados, debido a la escasa información estadística obtenida sobre volúmenes reales de captura en todos los años considerados.

\section{1 Extracción}

\section{Artes de pesca}

Para la captura de peces ornamentales se utilizan diferentes tipos de artes de pesca. En primer lugar, se encuentran las redes o paños con abertura de malla pequeña $(02-1.2 \mathrm{~cm})$ y Iongitud variable. Existe, además, las pusahuas, que tienen la forma de un cono truncado, cuyas partes la conforman un aro metálico de diferentes diámetros, equipado con una bolsa de malla menuda $(0.2-1.0 \mathrm{~cm})$ y con un mango de madera o aluminio. A simismo, la tarrafa 0 atarraya, diseñada para capturar peces de fondo y que se construye 
con un paño de malla con características similares a las ya mencionadas. La forma de operar varía de acuerdo a la destreza de cada pescador y a la disponibilidad o distribución de las especies. U na red arrastradora o de encierre necesita ser manipulada por tres personas y dos canoas pequeñas. Un pescador o "boyero", permanece en una de las canoas asegurando el cabo de uno de los extremos de la red, mientras los otros (shumbero), van soltando el paño con rapidez, realizando el encierro respectivo; al fina, ambos extremos coinciden y proceden a recoger la captura desde la canoa de mayor tamaño. La pusahua y la tarrafa son operadas por una sola persona y van acompañadas por una boga 0 remero de los ríos, cochas y quebradas, aprovechando las épocas de mayor abundancia.

\section{- Pescadores}

Existen dos tipos de extractores de peces en nuestra amazonía: los habilitados y los temporales u ocasionales. Los primeros son personas dedicadas a la pesca a tiempo completa; utilizan embarcaciones y motores obtenidos a través del préstamo otorgado por los exportadores, con el compromiso de cancelarlo en forma gradual con el producto. Por lo general, contratan otros pescadores para realizar las faenas.

Los pescadores temporales tienen por actividad permanente la agricultura y/o pesca de consumo y debido a circunstancias favorables, consiguen capturar algunas variedades de peces para acuario, almacenándolos en envases, muchas veces inadecuados, permaneciendo días en espera de los corredores para su comercialización.

\section{- Epocas de pesca}

L as actividades pesqueras se realizan durante gran parte del año. En época de vaciante, las aguas de los ríos se retraen y abandonan las zonas inundadas, facilitándosela captura, debido a que los peces son más vulnerables a las artes de pesca, al disponer de menores áreas de dispersión y protección. Ocurre lo contrario en creciente, cuando son inundadas grandes extensiones de la floresta amazónica.

Esta diferencia en el nivel de las aguas se produce en forma progresiva y escalonada a lo largo y ancho de los ríos principales. Esta particularidad es aprovechada por los pescadores para realizar las faenas de pesca, desplazándose de un lugar a otro, cuando las condiciones del río y la abundancia de peces no son adecuadas.

\section{Zonas de pesca}

La captura de los peces ornamentales que sustentan la exportación a nivel nacional e internacional se realiza en la mayoria de los cuerpos de agua de la 
red hidrográfica de la amazonía peruana; sin embargo, existen ríos, quebradas y cochas que aportan con mayor frecuencia y en cantidades superiores que otras. Entre estos últimos se tiene 1os siguientes sistemas fluviales

\begin{tabular}{|l|l|l|l|l|}
\hline \multicolumn{5}{|c|}{ SISTEM A FLUVIALES } \\
\hline RIO NAPO & RIO TIGRE & RIO UCAYALI & RIO AM AZONAS & RIO NANAY \\
\hline Río M azán & Queb. Choroyacu & Río Tapiche & Río A mpiyacu & Peña cocha \\
Río & Queb. Nahuapa & Río Blanco & Queb.Y acarite & Cocha Ilanchama \\
Tacshacuraray & Queb. Tigrillo & Cocha & Queb. Cojocma & Cocha Puñuisiqui \\
Río Curaray & Queb. Tigre & Chingana & Caño & Cocha Bravo \\
Río Tamboryacu & Queb. Huagana & Cocha Flor & Caballo cocha & Cocha Diamante \\
Cocha Zapote & & de castaña & & Cocha Samito \\
A tún cocha & Cocha & & \\
Que. Yanayacu & Huaracay & & \\
\hline
\end{tabular}

\section{- $\quad$ Especies capturadas}

En la captura y comercialización de peces se emplea con frecuencia el nombre comercial para identificar a una determinada especie 0 variedad. No se tiene referencia de la existencia de una lista o clasificación sistemática específica para peces ornamentales de la amazonia peruana. Los trabajos de Fowler (1945 - 1954), Axelrod (1981 - 1985) y Ortega - Van (1986), clasifican en forma general a los peces de aguas continentales.

Como aporte para dar inicio a un estudio más completo sobre taxonomía, se presenta a continuación una lista preliminar, ordenada por familias, géneros, especies y nombres comerciales de los peces que sustentan nuestra pesquería ornamental. 


\section{LISTA PRELIMINAR DE PECES ORNAMENTALES INDICANDO FAMILIA, NOMBRE CIENTIFICO Y NOMBRE COMERCIAL}

Familia / N ombre Preliminar

POTAMOTRYGONIDAE

1. Potamotrygon Ssp

LEPIDOSIRENIDAE

2. Lepidosiren paradoxa

OSTEOGLOSSIDAE

3. Osteoglossum bicirrhosum

CHARACIDAE

4. A cestrorhynchus ssp

5. A phyocharax

6. Boehkea fredcochui

7. Chalceus ssp.

8. Charaddium fasciatus

9. Hemigramus ocellifer

10. Hemigramus pulcher

11. Hemigramus sp.

12. Hyphessobrycon loretoensis

13. Hyphessobrycon bentoni

14. Hpessobrycon erythostigma

15. Hyphessobrycon sp.

16. M etynnis hypsauchen

17. Hyphessobrycon sp.

18. Myleus rubripinnis

19. M oenchausia oligolopis

20. M oenkhausia lepidura

21. Paracheirodon innesi

22. Priartobrama flligera

23. Serrasalmus spp.

24. Thayeria obligua

25. Triportheus rotundatus

GASITEROPELECIDAE

26. Carnegiella strigata

27. Carnegiella marthae

28. Torococharax stellatus
Nombre Comercial

Stinger ray

L ungfish

A rahuana

Cachorro

Cola roja

Tetra azul

Characidium

Hemigramus

Rodostumus

Tetra loreto

Rosaceus

Bleeding heart

Black ulrey

M etynnis

Roberty tetra

Red hook

M oenkhausia

A rgentino tetra

Neon tetra

Boodfin amazon

Piraña

Oblicua

Catalina

Hachet strigata

Hachet marthae

Hachet toracocharax 
HEM IODONTIDAE

29. Hemiodus sp.

CTEN OLUCIDAE

30. Boulengerella maculata

Beedle flsh

LEBIADINIDAE

31. Copeina guttata

32. Copella spp.

Copeina gutata

Copeina auratus

33. Nannostomus spp.

34. Nannostomus trifasciatus

Copema auratus

Trifasciatus

PARADONTIDAE

35. A pareidon pongoensis $\quad$ Pongo pongo

PROCHIL ODONTIDAE

36. Semprochilodus amazonensis Y araquí

CURIMATIDAE

37. Curimata vittata

Ractofogón

ANOSTOMIDAE

38. A bramites hypelonotus

A bramites

39. A nostomus anostomus

A nostomus

40. Leporinus friderici

L eporinus friderici

41. Leporinus fasciatus

L eporinus fasciatus

CHILODONTIDAE

42. Chilodus punctatus

Chilodus

GYMNOTIDAE
43. Eigenmannia sp.
Green knife fish

ELECTROPHORIDAE

44. Electmphorus electricus Electric eel

STERN OPY GIDAE

45. Sternophygus macrurus Ghost knife fish

HY POPOM IDAE

46. Hypopomus spp,

Spoted knife fish

RHAMPHIGTHYDAE

47. Rhamphichthys rostratus Elephant nose

DORADIDAE

48. Agamyxis pectinifrons $\quad$ Rafael spotted 
49. A mblydoras hancockii

50. Hassar spp.

51. M egalodoras irwini

52. Oxydoras niger

53. Platydoras costatus

AUCHENIPTERIDAE

54. Auchenipterichthys thoracatus

55. Parauchenipterus galiatus

ASPRENDINIDAE

56. Bunocephalus spp.

\section{PIMELODIDAE}

57. Brachiplatystoma filamentosum

58. Brachiplatystoma juruense

59. Brachiplatystoma sp.

60. Callopphysus macropterus

61. Duopalatinus sp.

62 Hemisorubin platyrhinchos

63. Leiarius marmoratus

64. M icroglanis sp.

65. Phractocephalus hemioliopterus

66. Pimelodus pictus

67. Pimelodus spp.

68. Pinirampus pirinampus

69. Paltystoma matichthys sturio

70. Platysilurus barbatus

71. Pseudoplatystoma fasciatum

72. Pseudoplatystoma tigrinus

73. Sorubirn lima

74. Sorubimichthys planiceps

\section{CALLICHTHYDAE}

$75 \quad$ Brochis splendens

76. Corydoras arcuatus

77. Corydoras agassizi

78. Corydoras elegans

79. Corydoras hastatus

80. Corydoras julii

81. Corydoras punctatus

82. Corydoras rabauti

83. Corydoras melanistus

84. Corydoras sp.

85. Corydoras sp.
Spinosissimus

Bufeo cunshi

Zuntarito churero

Oxidoras niger

Rafael stripped

Zamora cunchi

N ovia cunchi

Banjo cat

Zúngaro Dorado

Zúngaro alianza

Zúngaro saltón

Zúngaro mota

Cunshi blanco

Toa

A chara

Bumple bee cat

Red tailed cat (torre)

Pimelodella angelica

Pimelodella común

L ong finned rat

Zúngaro toa

Zungarito barbatus

Zúngaro doncella

Zúngaro tigre

Shovelnose (shiripira)

A cha cubo

Cory green cat

Cory arcuatus

Cory agassizi

Cory elegans

Cory hastatus

Cory julii

Cory puntatus

Cory rabauti

Cory melanistius

Cory San Juan

Cory orange cat 
86. Dyanema longibarbis

87 Dianema urostriata

LORICARIIDAE

88. Farlowella spp.

89. Loricaria spp.

90. Otocindus spp.

91. Hypostomus sp.

92. Chaetostomus spp.

RIVULIDAE

93. Pterolevias peruensi

94. Rivolus spp.

SY NBRANCHIDAE

95. Synbranchus marmoratus

NANDIDA

96. M onocirrhus ol acanthus

CICHLIDAE

97. A equidens spp.

98. A pistogramm agazzizi

99. A pistogramma spp.

100 Crenicichla spp.

101. Heros severum

102. M esonauta festivus

103. Pterophyllura scalare

104. Satanopera jurupari

105. Symphysodon aeguifasciatus

SOLEIDAE

106. Achirus achirus

TETRADONTIDAE

107 Colomesus osellus
Porthol corriente

Porthol rayado

Farlowella

L oricaria

Otocinclus

Plecostomus

X enocara

Rivolus peruensi

Rivulus común

A tinga, M arbled Eal

L eaft fish

A equidens moroni

A pistograma

A pistograma

A ñashúa

Severum

Festivum

Scalare

Jurupari

Discus

Sun fish

Puffers 


\subsection{Volumen de extracción}

La pesca se realiza en forma totalmente extractiva; desde el inicio se ha capturado sin tener en cuenta la magnitud del recurso y sin considerar que una pesca indiscriminada tendría efectos perjudiciales sobre las poblaciones ícticas existentes. La presión de pesca ejercida sobre el stock disponible ha ido incrementándose con el tiempo, originada por un aumento en el número de empresas exportadoras y, por consiguiente, de pescadores. Por el contrario, los volúmenes de exportación totales por especie, han disminuido significativamente, como lo demuestran las estadísticas obtenidas (Cuadro N2 1). Esta situación, aunada a la competencia de países vecinos (Brasil y Colombia) y a la reproducción masiva de algunas especies en cautiverio, ha ocasionado una fuerte caída de los volúmenes comercializados en los últimos años. En 1978, el Perú exportó 19'581,539 peces, que generaron un total de US\$ 878,000. Desde aquel entonces, ha disminuido hasta alcanzar los 5939,771 de ejemplares en 1988, produciendo ese año un ingreso por concepto de divisas del orden de US $\$ 670,000$ (Cuadro N 23 ).

Del análisis por especies, se determinó que la familia CALLICHTHYDAE, con el género CORYDORAS, ocupó el primer lugar en cuanto a número de ejemplares exportados y divisas generadas, con 4539,029 unidades y US\$ 312,225 en el año 1977, hasta descender a 1461,975 ejemplares y U S\$ 102, 012 en 1988. Sigue en orden de importancia la familia Characidae, con Paracheirodon innesi - neon tetra, como la especie más representativa, con

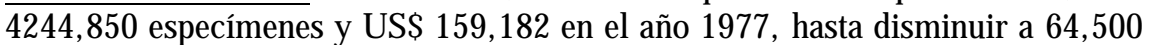
el número de peces exportados y producir ingresos por US\$2,419 en el último año. Este pronunciado descenso del volumen de exportación se debe a que Taiwán y Japón, están reproduciendo esta variedad de neón en cautiverio y lo exportan a precios por debajo de los ofertados por los países extractores tradicionales. (Presidente AEDO, com pers.) 


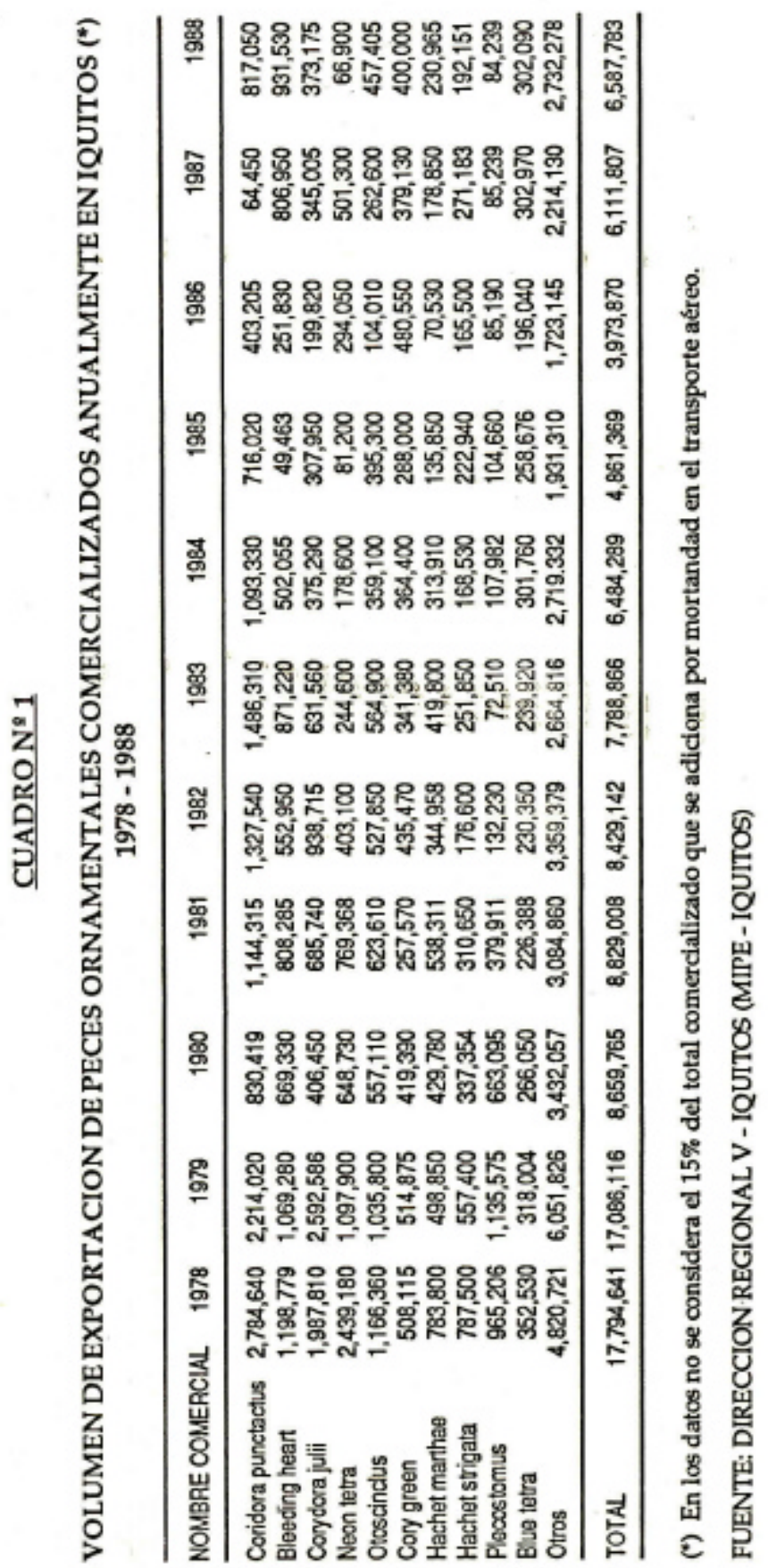




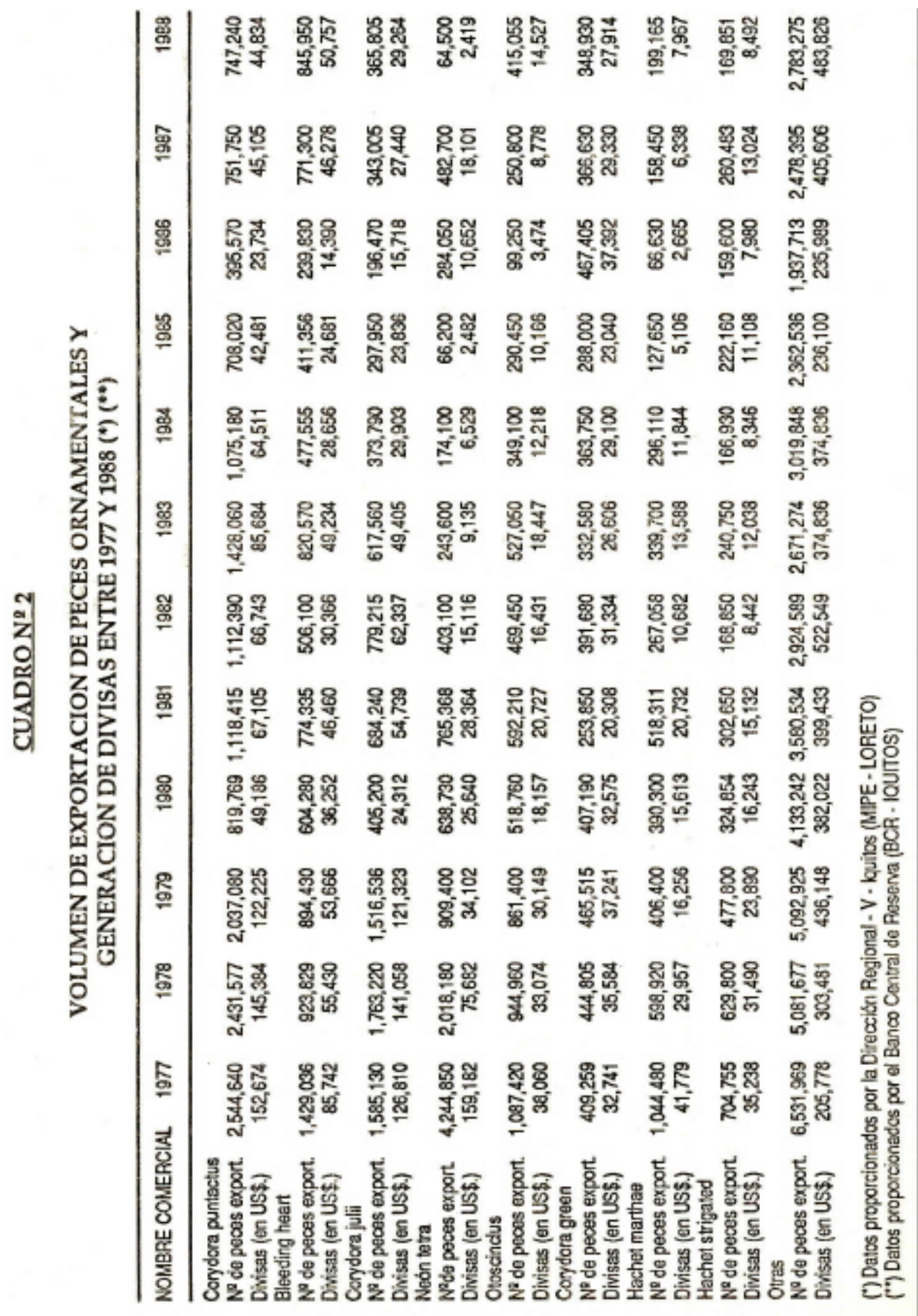


El tercer lugar lo ocupé la familia Gasteropelecidae, con el género Carnegiella (Hachet). Como el más importante, con 1'749,235 unidades, generando la cantidad de US\$77,017 en el año 1977 hasta llegar al número de peces comercializados a 369,016 y el monto generado en el último año a U S\$16,459. La familia Loricariidae, con el género Otocinclus, ocupa el cuarto lugar en cuanto a contribución por número de especímenes, con 1087,420 para el año 1977 y 45,066 para el último año, habiendo generado ingresos por concepto de divisas del orden de los US $\$ 38,060$ y US $\$ 14,527$, respectivamente.

En los Cuadros $\mathrm{N} N 2$ y 3 , se observa el comportamiento histórico de los volúmenes exportados y la generación de divisas en los últimos 11 años, en forma general y por especies. Se nota una leve recuperación en 1981, para continuar la tendencia negativa hasta alcanzar en 1986 el punto más bajo de la comercialización con 3.8 millones. En los dos últimos años, se produce un pequeño incremento, pero muy por debajo del promedio anual comercializado. Esta variación lo explica Russel (1931 - 1942), citado por Wosnitza (1978), en su teoría de la sobrepesca, donde menciona la existencia de una gran influencia de la pesca sobre la estructura poblacional de los peces. Indica también que, con el aumento de la captura (sobrepesca), disminuye la captura por unidad de esfuerzo (densidad del stock) y disminuye el rendimiento después de un aumento inicial del volumen de captura.

En la información estadística analizada (Cuadro № 3) se aprecia el incremento de los volúmenes de las exportaciones de peces de consumo humano comercializados como ornamentales. Los precios of ertados por estas especies de alto valor nutritivo para el poblador amazónico, son atractivos y la demanda de los países importadores va en aumento; razón por la cual, las capturas se efectúan con mayor frecuencia. D entro de ellas se encuentran los grande bagres o zúngaros que, durante la primera etapa de su vida, son muy apreciados por Ios acuaristas. En 1976, se comercializaron un total de 4,853 ejemplares de estos bagres, el último año de exportación alcanzó la suma de 13,438 unidades, que generaron divisas por un monto de US\$35,533 y US\$ 67,190, respectivamente.

A continuación, se presenta una relación se especies consideradas dentro del grupo genérico de zúngaros:

D oncella (Pseudoplatystoma fasciatum)

Tigre zúngaro (Pseudoplatvstoma tigrinum)

D orado (Brachiplatystoma filamentosum)

Saltón (Brachiplatystoma spp)

A chacubo (Sorobimichthya lanices

Shiripira Sorubin lima 


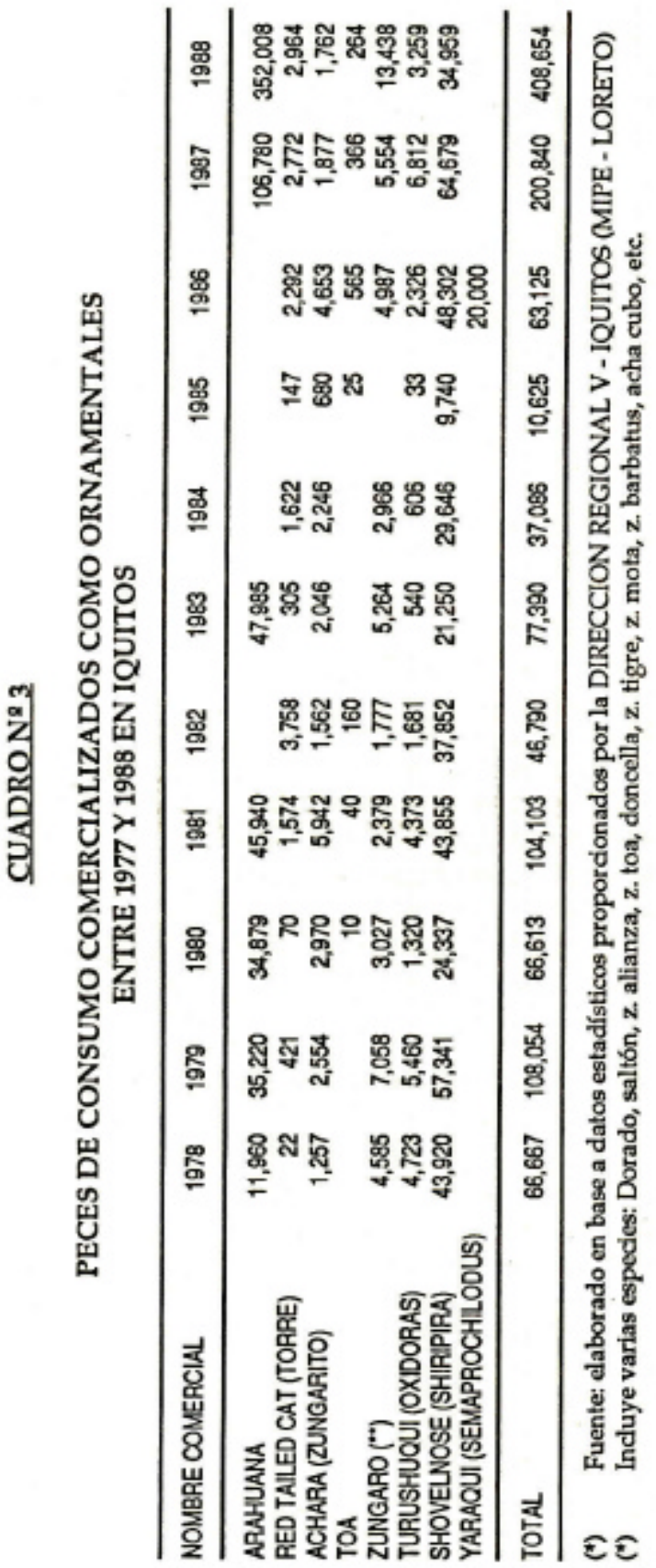


La shiripira o shovelnose (sorubim lima), es una especie de gran demanda en el mercado de peces de consumo, por la calidad de su carne. En 1978, se exportaron 43,920 ejemplares, para alcanzar en 1987 la cantidad de 64,679 unidades. Algo similar está ocurriendo con la arahuana (Osteoglossum bicirrhosum), en el año 1978 se exportaron I1, \%0 especimenes y en el último año la comercialización de esta especie alcanzó la cifra de 352,000 ejemplares; logrando producir ingresos por concepto de divisas del orden de US\$ 8,372 y US\$ 246,405, respectivamente. Existen dispositivos legales por los cuales se prohíbe la extracción como ornamentales de algunas especies de consumo humano, no figurando ninguna de las mencionadas anteriormente.

Dispositivos legal es vigentes:

1. D.L. № 18810, aprueba la L ey General de Pesquería.

2. D.S. № 011-71-PB, Reglamento de la L ey General de Pesquería.

3. Resolución M injsterial $\mathrm{N} \cong$ 0011-72-PE, dél 12.01.72. Prohibela extracción como ornamentales de las especies ictiológicas destinadas al consumo humano de la Amazonla Peruana, excepto las especies "arahuana", acarahuazú', "boquichico y "palometa".

4. Resolución Ministerial $\quad \mathrm{N} 0$ 00298-76-PB, del 21.11.76, prohibe lá extracción para ser comercializada como peces ornamentales las especies "boquichico" (Prochilodus sp), "arahuaca (Osteoglossum bicirrhosum), "acarahuazú" (A stronotus ocellatus) y "palometa" M ylossoma sp. J. De la misma manera, deroga la R.M. № 0011-72PE.

5. Resolución M inisterial NI2 029-79-PB, 07.09.79, modifica el artículo 10 de la R.M. N 02 0898-76-PB, exceptuando de sus alcances ala especie denominada "arahuana" (Osteoglossum bicirrhosum).

6. Decreto Supremo $N 02$ 005-84--PE, aprueba el reglamento para la extracción y comercialización de recursos hidrobiológicos ornamentales y en su artículo 50 especifica claramente que el Ministerio de Pesquería establecerá como parte de sus funciones, Io siguiente:

a. Las especies cuya extracción está prohibida o restringida.

b. Volumen máximo de captura.

c. Zonas y épocas de veda.

d. Equipos y métodos de captura.

e. $\quad L$ as acciones necesarias para la conservación de las especies.

7. Resolución Directoral N2023-88-PE/DIREPE-V-Iquitos, del 18.03.88, prohibe la extracción para ser comercializados con fines ornamentales los alevinos de las especies (Colossoma macropomum) "gamitana, (Piaractus brachypomus) "paco", (Bryon erythropterum) "sábalo cola roja', (Brycon melanopterum) "sábalo cola negra" y (Cichla ocellaris) "tucunaré". 
8 Aparte, existe una disposición especial para el "paiche' A rapaima gjgas. Especie que según la legislación vigente de la pesquería continental, está protegida por una veda para favorecer el desove y crecimiento inicial de los alevinos y con la R.M. 85-PE. Del 25.07.85, prohibe la captura de ejemplares menores de 1.60 de longitud total en todos los recursos hídricos del país.

De las normas legales existentes, se determina que las especies de consumo humano prohibidas para la exportación son las siguientes:
A carahuazü
Boquichico
(A stronotus ocellatus)
Gamitana
(Prochilodus nigricans)
Paco
Paiche
(Colossoma macropcrnttm)
(Piaractus brachvpornum)
Sábalo cola roja
(A rapaima gigas)
Sábalo cola negra
(Brycon ervthropL erum)
Palometa
(Brvcon melanopterum)
Tucunaré
(M ylossoma sp.)
(Cíchla ocellaris)

A simismo, las Resoluciones $M$ inisteriales son contradictorias, mientras que por la R.M. 001 1-72-PE, se prohibe la captura como peces ornamentales de las especies ictiológicas destinadas para consumo humano, exceptuando cuatro (04) especies, sin criterio técnico que puede sustentar esta acción; además, la misma redacción de las resoluciones no es clara y crea confusión.

El caso de la especie A rahuanaes especial, con la R.M. 00172-PE., se autoriza su comercialización, con la R.M. 029-76-PE, se prohibe su captura como ornamental y con la R.M. 029-79-PE, se vuelve a autorizar su comercialización. Considerando que en la selva baja existen unas 50 especies de consumo humano directo, yque sus poblaciones podrían verse afectadas de continuar su comercialización como ornamentales, en su estado de alevinos, se determina que los dispositivos legales vigentes son insuficientes y, por consiguiente, sólo protegen a un $20 \%$ de las especies Icticas que sustentan la alimentación del poblador amazónico.

4.3 Transporte y comercialización. Luego de la captura, los peces son transportados por los pescadores, utilizando bolsa plásticas, cajas de material sintético, bidones cortados, e inclusive, dentro de canoas llenas de agua. Con mucha frecuencia esperan la llegada de los corredores para efectuar la venta muy cerca a la zona de pesca.

Los corredores son empleados o comisionados que trabajan para las empresas exportadoras y se movilizan en potentes botes deslizadores, a lo largo de los principales ríos, dedicándose a comprar las capturas de los pescadores ocasionales y de los grupos dedicados a esta actividad. Transportan el producto 
en cajas de plástico y/o en bolsas del mismo material, colocados dentro de cajas de madera (triplay).

Los intermediarios o rematistas son personas encargadas por los exportadores para realizar las compras en los principales puertos de desembarque, haciéndolo, por lo general, a precios por debajo de lo ofertado en el mercado.

Los precios que rigen la comercialización de los peces ornamentales, está de acuerdo a la ley de la oferta y la demanda; sin embargo, para la declaración del valor de la exportación, se toma como referencia la lista de precios elaborada años atrás por la DIREPE-V (Cuadro $\mathrm{N}$ 4), la cual no ha sido actualizada e incluye solamente el $65 \%$ de las especies ornamental es que se exportan.

Al ingresar al acuario, los peces son contados, clasificados y registrados en una ficha. Luego, son colocados en estanques de cemento, vidrio y/o madera, cubiertos de fibras sintéticas, para recibir el tratamiento profiláctico respectivo y estar a la espera de los trámites para el envío definitivo a los importadores.

Los requisitos para efectuar un embarque, son los siguientes:

- Permiso

- Declaración J urada

- Trámite en agencia de aduana

- Inspecciones

- Licencia de exportación

- Fianza bancaria

- Trámite de aduana

Para obtener el permiso previo, es necesario presentar una lista consignando especies y cantidades, la lista de peces ingresada a los acuarios está disponible en el área de extracción de la DIREPE V. Se tiene ciertas dudas acerca del grado de confiabilidad de esta información, debido a la existencia aparente de un millón de peces sin destino, registrados en el transcurso de 1988. Este resultado se ha obtenido por diferencia entre el número de ejemplares ingresados y el número exportado durante ese año, considerando que el porcentaje de mortalidad es el siguiente:

- Desde la captura hasta el ingreso 20\%

- Permanencia en el acuario 30\%

- Transporte aéreo 15\%

FUENTE: Informe técnico (1969)

Pescadores

DIREPE V - Exportadores 


\section{CUADRO № 4}

\section{LISTA DE PRECIOS MINIMOS DE PECES ORNAMENTALES POR ESPECIE}

\begin{tabular}{lc}
\hline N OM BRE COM ERCIAL & $\begin{array}{c}\text { PRECIO EXPORTACION (US\$) } \\
\text { (VALOR DECLARAD O) }\end{array}$ \\
\hline A bramites & 0.30 \\
A chara & 3,0 \\
A pistograma & 0.05 \\
A rahuana & 0.70 \\
A uratus & 0.05 \\
A tinga común & 1.00 \\
Black ulrey & 0.04 \\
Bleeding heart & 0.06 \\
Blood fish amazon & 0.04 \\
Blue tetra & 0.06 \\
Bumple bee cat & 017 \\
Chalceus & 0,35 \\
Characidium fasciatus & 0.04 \\
Chilodus punctatus & 0.08 \\
Copeina arnoldi & 0.04 \\
Copeina crenimaculate & 0.10 \\
Copeina four sport & 0.15 \\
Co.peina redstrip & 0.03 \\
Cory agazzizi & 0.06 \\
Cory arcuatus & 0.08 \\
Cory elegans & 0.05 \\
Cory green cat & 0.08 \\
Cory hastatus & 0.03 \\
Cory julii & 0.08 \\
Cory melnistius & 0.05 \\
Cory punctatus & 0.06 \\
Cory rabauti & 0.08 \\
Cory San Juan & 0.05 \\
Dávila tetra & 0.08 \\
Discus & 2.25 \\
Domino tetra & 0.05 \\
Electril eel & 5.00 \\
Elephant nose & 100 \\
Farlowella & 0.15 \\
Festivum & 0.06 \\
Glass tetra & 0.10 \\
Hachet strigata & 0.05 \\
M etynis & 0.50 \\
&
\end{tabular}


M oenkhuasia

$\mathrm{N}$ eon tetra

N ovia cunchi

Oblicuas

Ocelifer

0.04

Otoncinclus

0.035

Orange cat

0.08

Pimelodella angelica

020

Pimelodeila común

Plecostomus

Porthol rayado

Porthol corriente

0.05

Rafael stripped

0.25

Rafael spoted

Red tailed cat (torre)

Rivelus peruensi

Rosaceus

0.03

Scalare

0.10

Severum

Spinosissmus

Stringer ray

Trifasciatus

Y ellow cat

Zamora ci.mchi

Zungarito achacubo

Zungarito alianza

Zungarito dorado

Zungarito tigre

Para embalar los peces se utilizan bolsas de polietileno de 4-5 It. De capacidad, Ilenos de agua, previamente tratada. EI número de ejemplares varía, dependiendo de la distancia a recorrer, la especie y el tamaño. A ntes de cerrar la bolsa, se inyecta oxígeno o aire para evitar la muerte de los peces durante el transporte. L uego de cerrarla, con una banda de goma son depositados en cajas de cartón o madera (triplay). 
Los costos por conceptos de transporte son cubiertos íntegramente por los importadores, realizándose los despachos con la modalidad de contraentrega.

Los canales de comercialización y distribución de peces ornamentales se esquematizan en el Cuadro $\mathrm{N} 0$ 5. Se observa que Iquitos es la principal fuente de abastecimiento del mercado nacional e internacional.

Los EE.UU. absorben el $88.5 \%$ de la producción anual de esta zona, siendo los importadores de Miami los principales clientes. U na pequeña parte (13\%) es enviada a Lima para cubrir el mercado nacional e indirectamente abastecer a Europa, Asia y Latinoamérica. Pucallpa contribuye destinando el $98 \%$ de su producción a lquitos y el $2 \%$ a Lima. Los volúmenes capturados en la zona fronteriza de Caballo Cocha, son comercializados mayormente en Leticia (Colombia), ocasionando una fuga de divisas hacia el país vecino.

Actualmente, en Iquitos, operan cerca de 30 empresas dedicadas a la exportación de peces ornamentales. Todas debieron cumplir con los requisitos solicitados en el Reglamento para la Extracción y Comercialización de Recursos Hidrobiológicos Ornamentales (D.S. № 005-84-PF). Cada una de ellas cuenta con personal de planta y apoyo, calculándose en 5,000 personas, que dependen directamente e indirectamente de esta actividad. En el Cuadro $N 05$, se presenta una relación de los exportadores e importadores registrados a diciembre de 1988.

\section{CUADRO № 5 \\ RELACION DE EXPORTADORES E IMPORTADORES DE PECES ORNAMENTALES}

\begin{tabular}{|c|c|c|c|c|}
\hline A cuario Suramérica & Iquitos - & Perú & River Trading Co. & California - USA \\
\hline Peces de la A mazonia & $"$ & $"$ & Fast East Enterprise & N orita - Japón \\
\hline Corpez & $"$ & $"$ & .C. Tropical & M iami - USA \\
\hline A cuario M oronacocha & $"$ & $"$ & L ebaco Enterprise & Miami - USA \\
\hline Blue A cuario S.C.R.L. & $"$ & $"$ & Ruinemans A cuarium & Miami - USA \\
\hline A cuario Tropical & $"$ & $"$ & Jull Chine Traders & Taiwan - China \\
\hline A cuario U niversal & $"$ & $"$ & Reinal do Pines & California - USA \\
\hline Y acuruna A quarium & $"$ & $"$ & San W ood Fisheries & Hon Kong - China \\
\hline Ruíz E. Dávila & $"$ & $"$ & A quatina Taypes & Taiwan - China \\
\hline Iquitos Fish E.I.R.L. & $"$ & $"$ & A. Salazar Import - & Ex M iami - USA \\
\hline E. Bustamante & $"$ & $"$ & A cuario Enterprise & Miami - USA \\
\hline A cuario Itaya & $"$ & $"$ & A cuatic Tranship & Miami - USA \\
\hline CRIAPEZ & $"$ & $"$ & Julio Documett & Miami - USA \\
\hline Piscis A quarium & $"$ & $"$ & A cuario W ang & Miami - USA \\
\hline A cuario A mazonas & $"$ & $"$ & Colunesian Tropical & California - USA \\
\hline J ungle Sports & $"$ & $"$ & Nanshow Co. & Taiwan - China \\
\hline Tropical Fish A quarium & $"$ & $"$ & \multicolumn{2}{|c|}{ A quapex Corpi M iami - USA } \\
\hline
\end{tabular}




\begin{tabular}{|c|c|c|c|}
\hline San J udas Tadeo S.C.R.L & $"$ & " A quatic Blékers & M iami-USA \\
\hline A cuario Peas S.A. & $"$ & Suriname Tropical Fish & Miami-USA \\
\hline T. Ochoa de M éndez & " & " Chi Hai Trading CEFP & Taiwa-China \\
\hline Iquitos Fishery E.I.R.L & $"$ & " T.H. Ruine M an's & M iami-USA \\
\hline Perú Fish S.C.R.L. & $"$ & " Dolphin INT'L & California - USA \\
\hline Stern Fish A quaríum & $"$ & M.E. Sánchez & California - USA \\
\hline A cuario Chullachaqui & $"$ & Keny Trading & California - USA \\
\hline A cuario Río Blanco & $"$ & Essington A quatica & L ondon- Inglaterra \\
\hline A quario Delfín & $"$ & Rainbow Tropical & Miami - USA \\
\hline 1. L. Sáenz & $"$ & Gulf Coast A cuatic & Miami - USA \\
\hline A cuario Granja A zul & $"$ & A rbufish aq. & Miami - USA \\
\hline A cuario Continental & $"$ & " A rbufish aq. & \\
\hline A cuario & Lima & Lima-Perú M iami DADE Fisch & Miami USA \\
\hline U niversal Trading & u & " ERDA Inc. & M iami - USA \\
\hline Paflet S.A. & $"$ & O ceanographer Aq. & Japón \\
\hline
\end{tabular}

Fuente: DIREPE V - Iquitos

\section{CONCLUSIONES}

- De continuar esta situación, las poblaciones de peces ornamentales podrían verse afectadas por una fuerte presión de pesca y llegar a un punto en el que la densidad del recurso disminuya significativamente hasta ocasionar el colapso de la pesquería.

- Las cifras referentes a volúmenes de captura, ingreso de peces a los acuarios, número de peces exportados y por, consecuencia, de valores económicos, no son confiables.

- Se están exportando peces utilizados para el consumo humano como ornamentales.

- Los dispositivos legales existentes son insuficientes y en algunos casos contradictorios.

\section{RECOMENDACIONES}

Diseñar e implementar, en forma inmediata, un sistema de registro de datos estadísticos para todas las etapas de la explotación de peces ornamentales y en forma paralela, como medida cautelatoria del recurso, se sugiere el establecimiento de cuotas de exportación no mayores a los volúmenes comercializados en 1988. 
Efectuar un estudio, que podría hacerse a través de un convenio entre el MIPE Exportadores - IIAP, sobre taxonomía, bioecología, potencial íctico y reproducción en cautiverio de las principales especies de peces ornamentales.

Restringir la exportación de peces de consumo humano considerados como ornamentales por medio de dispositivos legales claros y precisos.

Coordinar con los países miembros del Tratado de Cooperación A mazónica, con el fin de establecer un sistema legislativo y comercial adecuado para salvaguardar los intereses comunes de las naciones exportadoras de peces ornamentales.

\section{BIBLIOGRAFIA}

1. Axelrdo, H. et al (1980). Exotic Tropical Fish, Expanded Edition. T.EH. Publications Inc. N ew Y ork. USA. 1,300 pág.

2. Axelrdo, H. Et al (1980). A tlas of Freshwater Acuarium FishesT.F.H. Publications Inc. N ew Y ork - USA. 780 pág.

3. Bonetto, A. (1981). Informe relativo abs estudios limnológicos a realizar con la A mazonía Peruana. Proyecto UNDP/FAO-PER/76/022. Instituto del Mar del Perú. Informe NI2 81. Lima - Perú. P.P. 173-205.

4. Filomeno, Ponce del Prado, Castañeda y M ejía (1969). Informe de la Comisión designada por R.S. NI2 15-69-A G-DP, para el estudio de la comercialización, exportación y gravámenes de los peces ornamentales, animales vivos y plantas acuáticas de la amazonia.

5. Fowler, H. (1945). Los peces del Perú, Catálogo sistemático de los peces que habitan en aguas peruanas. Lima. M useo de Historia Natural 'Javier Prado'. 298 pág.

6. Fowler, H. (1945). Os Peixes de Agua Doce do Brazil, 4ta. Entrega. A rquivos de zoología, Sao Paolo (9) 1-400.

7. Montreuil, Castañeda, Rodríguez, Pezo, Cruz (1984). Diagnóstico de la pesquería en la Región A mazónica. U cayali. IIAP. 126 pág.

8. MontreuilV.H. (1988). Explotación de larvas yalevinosde arahuana (Osteoglossum bicirrhowum) como especie ornamental IIAP, Informe Técnico.

9. Ortega, NI. Y Van, R. (1986). A nnotated cheddist of the ftes water fishes of Perú. Smithsonian contribution to zoology; NI2 437.25 pag.

10. Welcome, R.L. (1975). The fisheries ecology of A frican Food plains. FAO cifa. TECH. PAPER 3-ROME - ITALY. 
11. WOSNITZA, C. (1978). M anual de dinámica de poblaciones de peces. Trujillo, Perú. U niversidad Nacional de Trujillo. IIAP. 111 pág. 